\title{
X-linked inheritance of Fanconi anemia complementation group B
}

\author{
Amom Ruhikanta Meetei ${ }^{1,5}$, Marieke Levitus ${ }^{2,5}$, Yutong Xue ${ }^{1}$, Annette L Medhurst ${ }^{2}$, Michel Zwaan $^{3}$, \\ Chen Ling ${ }^{1}$, Martin A Rooimans ${ }^{2}$, Patrick Bier ${ }^{2}$, Maureen Hoatlin ${ }^{4}$, Gerard Pals ${ }^{2}$, Johan P de Winter ${ }^{2}$, \\ Weidong Wang ${ }^{1} \&$ Hans Joenje ${ }^{2}$
}

Fanconi anemia is an autosomal recessive syndrome characterized by diverse clinical symptoms, hypersensitivity to DNA crosslinking agents, chromosomal instability and susceptibility to cancer ${ }^{1,2}$. Fanconi anemia has at least 11 complementation groups $(A, B, C, D 1, D 2, E, F, G, I, ~ J, ~ L))^{3,4}$; the genes mutated in 8 of these have been identified. The gene BRCA2 was suggested to underlie complementation group $B$, but the evidence is inconclusive ${ }^{5}$. Here we show that the protein defective in individuals with Fanconi anemia belonging to complementation group $B$ is an essential component of the nuclear protein 'core complex' responsible for monoubiquitination of FANCD2, a key event in the DNAdamage response pathway associated with Fanconi anemia and BRCA $^{3,6,7}$. Unexpectedly, the gene encoding this protein, $F A N C B$, is localized at Xp22.31 and subject to X-chromosome inactivation. $X$-linked inheritance has important consequences for genetic counseling of families with Fanconi anemia belonging to complementation group $B$. Its presence as a single active copy and essentiality for a functional Fanconi anemia-BRCA pathway make $F A N C B$ a potentially vulnerable component of the cellular machinery that maintains genomic integrity.

We previously purified a Fanconi anemia core complex containing five known Fanconi anemia proteins and four unknown components, called Fanconi anemia-associated polypeptides (FAAPs). We showed that FAAP43 (also called FANCL) is a ubiquitin ligase and was defective in an individual affected with Fanconi anemia belonging to a new complementation group (FA-L) ${ }^{3,7}$ (Fig. 1a). Here we report that FAAP95 (also called FAAP90) is mutant in individuals with Fanconi anemia belonging to complementation group B (FA-B).

Analysis by mass spectrometry identified FAAP95 as FLJ34064, a hypothetical protein of unknown function. A polyclonal antibody raised against FLJ34064 specifically recognized the 95-kDa polypeptide of the Fanconi anemia core complex immunopurified by an antibody to FANCA, indicating that FLJ34064 is FAAP95 (Fig. 1b). Immunoblotting also detected the presence of FAAP95 in Fanconi anemia core complex proteins immunoisolated by a Flag antibody from 293 cells stably expressing Flag-tagged FANCL ${ }^{7}$ (Fig. 1c). Reciprocal immunoprecipitation with the antibody to FAAP95 showed coprecipitation of several components of the Fanconi anemia core complex, such as FANCL, FANCA and FANCG (Fig. $\mathbf{1 b}, \mathbf{c}$ and data not shown). These data suggest that FAAP95 is a stable component of the Fanconi anemia core complex. We also found that FAAP95 coimmunoprecipitated much more FANCL than FANCA or FANCG, suggesting that the interaction between FAAP95 and FANCL might be direct.

Notably, depletion of FAAP95 reduced the amount of monoubiquitinated FANCD2 (Fig. 1d), similar to depletion of other components of the Fanconi anemia core complex ${ }^{7,8}$, indicating that FAAP95 is a functional component of this complex. In addition, cells depleted of FAAP95 had less FANCL, suggesting that FAAP95 is required for the stability of FANCL.

FAAP95 has sequence homologs in mouse and chicken, but not in Drosophila melanogaster or Caenorhabditis elegans. The $\mathrm{C}$ terminus of this protein contains a putative bipartite nuclear localization signal (NLS; Supplementary Fig. 1 online), and we found that most of the protein was present in the nuclear extract of HeLa cells (Fig. 2a). Notably, FAAP95 was retained mostly in the cytosolic extract of a FANCA-deficient cell line but became localized to the nuclear extract on complementation by wild-type FANCA (Fig. $2 \mathbf{b}$ and Supplementary Fig. 2 online). In this respect, FAAP95 is similar to $\mathrm{FANCL}^{7}$, suggesting that FAAP95 is associated with Fanconi anemia.

The gene encoding FAAP95 is localized on the $\mathrm{X}$ chromosome (Xp22.31); therefore, individuals affected with Fanconi anemia with mutations in this gene should all be males. Cells from such individuals should also show defective monoubiquitination of FANCD2 on the basis of the involvement of the core complex in this process and the results of our short interfering RNA (siRNA) experiments (Fig. 1d).

\footnotetext{
${ }^{1}$ Laboratory of Genetics, National Institute on Aging, National Institutes of Health, 333 Cassell Drive, TRIAD Center Room 3000, Baltimore, Maryland 21224, USA. ${ }^{2}$ Department of Clinical Genetics, VU University Medical Center, Van der Boechorststraat 7, 1081 BT Amsterdam, The Netherlands. ${ }^{3}$ Department of Pediatrics and Hematology/Oncology, VU University Medical Center, PO Box 7057, 1007 MB, Amsterdam, The Netherlands. ${ }^{4}$ Division of Molecular Medicine \& Molecular and Medical Genetics/Oregon Health and Science University, 3181 SW Sam Jackson Park Road, Portland, Oregon 97239, USA. ${ }^{5}$ These authors contributed equally to this work. Correspondence should be addressed to W.W. (wangw@grc.nia.nih.gov) or H.J. (joenje@vumc.nl).
} 
a

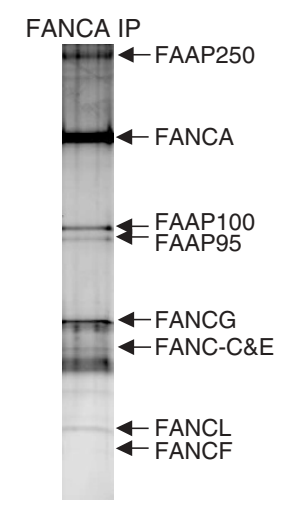

b

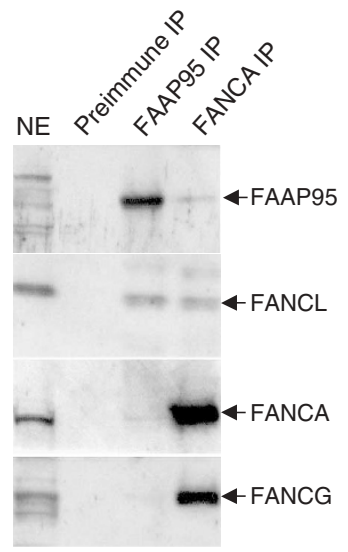

C

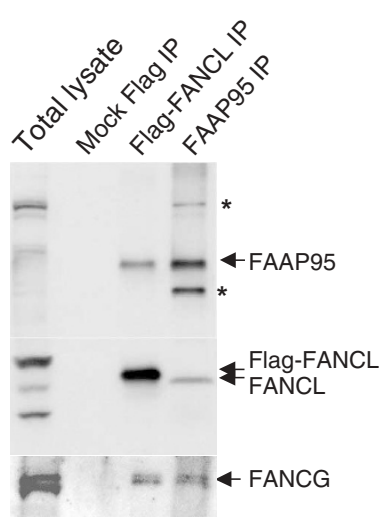

d

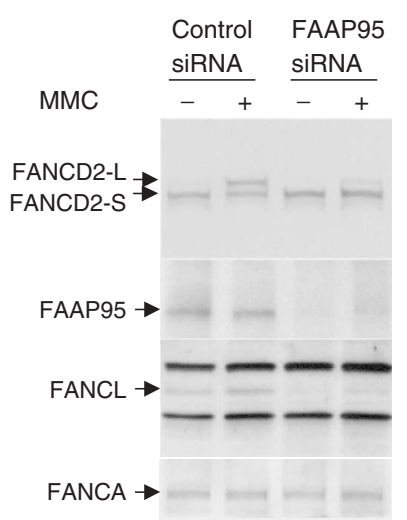

Figure 1 FANCB is a member of the core complex required for monoubiquitination of FANCD2. (a) Silver-stained SDS-PAGE gel showing the immunopurified Fanconi anemia core complex ${ }^{7}$. The polypeptide previously called FAAP90 is now called FAAP95 because its relative molecular mass is $95 \mathrm{kDa}$.

(b) Immunoblotting of polypeptides obtained from immunoprecipitation (IP) with antibodies to FANCA or FAAP95. Immunoblotting of the nuclear extract (NE) was included to show the correct size of the Fanconi anemia proteins (except FAAP95). (c) Immunoblotting of Fanconi anemia core complex proteins immunoisolated with a Flag antibody from 293 cells stably expressing Flag-FANCL. Immunoprecipitation by FAAP95 antibody was analyzed as a control. Asterisks indicate two nonspecific polypeptides that crossreact with antibody to FAAP95. (d) Immunoblotting of lysates of HeLa cells depleted of FAAP95 by siRNA. HeLa cells treated with a scrambled siRNA oligonucleotide were included as a control.

옹

Of the three Fanconi anemia complementation groups whose underlying genes have not been identified (FA-B, FA-I and FA-J), only individuals with FA-B (individuals HSC230 and EUFA178) met these two criteria. FAAP95 protein was undetectable in an analysis of lymphoblastoid cell lines from these individuals, indicating that the gene encoding this protein may be FANCB (Fig. 3a).

The sequence of the region encoding FAAP95 in HSC230 cells showed a frameshift mutation (1838insT) in exon 8, resulting in a premature stop codon upstream of the NLS consensus sequence (Fig. 3b). No cDNA could be amplified by RT-PCR from individual EUFA178, owing to a deletion of 3,314 bp (10693del3314) that (2) included the promoter region and exon 1. We selected two other individuals (individuals EUFA1082 and EUFA1386) as candidates for FA-B on the basis of their exclusion from most of the other complementation groups, their inability to monoubiquitinate FANCD2 and their male gender. We found frameshift mutations in both individuals, 1650delT (exon 8) in individual EUFA1082 and 811insT (exon 3) in individual EUFA1386 (Fig. 3b), both of which lead to early stop codons upstream of the NLS.

In addition, we transfected lymphoblasts from individuals with FA-B with cDNA encoding FAAP95 and found that the abnormal phenotypes associated with Fanconi anemia, hypersensitivity to mitomycin C (MMC) and monoubiquitination of FANCD2, were both restored to normal (Fig. 3c,d and Supplementary Fig. 3 online). These data proved that the gene encoding FAAP95 is the gene associated with FA-B; therefore, we named the gene FANCB.

Because X-linked inheritance has not been reported in Fanconi anemia, we tested the mothers and grandmothers of the affected males to determine whether they carried the mutations (see Fig. $\mathbf{4}$ for the pedigrees of the families studied). The mothers of individuals EUFA178 and EUFA1386, and a healthy sister of EUFA178, carried the mutations (Fig. 5). The mutations were not found in the grandmothers or mothers of individuals EUFA1082 and HSC230. These findings suggest that the mothers (of individuals HSC230 and EUFA1082) or a grandparent (of individuals EUFA178 and EUFA1386) may have been germline mosaics for these mutations. Alternatively, a de novo mutation may have occurred after fertilization in these individuals.

In adult females, most genes on one of the $\mathrm{X}$ chromosomes are inactive owing to hypermethylation of their promoter ${ }^{9}$. This $\mathrm{X}$ inactivation occurs early in embryogenesis and is randomly determined. Once inactivated, the inactive $\mathrm{X}$ remains clonally persistent ${ }^{10}$. As a result, $\sim 50 \%$ of cells in females have an active paternal $\mathrm{X}$ chromosome, whereas the other $50 \%$ have an active maternal $\mathrm{X}$ chromosome $\mathrm{e}^{11,12}$. However, FANCB is situated in a region where $\sim 60 \%$ of the genes escape inactivation and are expressed a

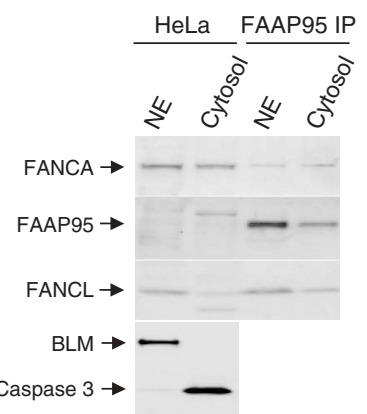

b

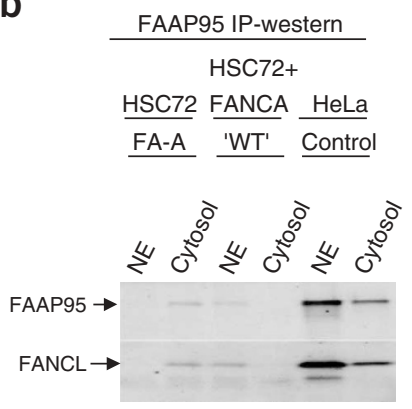

Figure 2 Nuclear localization of FAAP95 depends on FANCA.

(a) Immunoprecipitation (IP)-coupled western-blot analysis showing the presence of FAAP95 and its associated complex in both nuclear and cytosolic extracts of HeLa cells. The amount of FAAP95 is too low to detect by direct immunoblotting, unlike that of the other two Fanconi anemia proteins (FANCA and FANCL). BLM (a DNA helicase) and caspase 3 were analyzed as markers for the nuclear (NE) and cytosolic extracts, respectively. (b) Immunoprecipitation-coupled western-blot (IP-western) analysis showing the presence of FAAP95 and its associated FANCL in the cytosolic extract of a cell line defective in FANCA. On complementation of the same cell line by exogenous FANCA ('WT'), both proteins are detected in the nuclear extract (NE). HeLa cells were analyzed as a control. A control experiment is shown in Supplementary Figure 2 online. 
Figure 3 Evidence of a genetic defect in FAAP95 in individuals with FA-B. (a) Immunoprecipitation-coupled western-blot (IP-western) analysis showing the absence of FAAP95 in whole-cell lysates from two available cell lines from individuals with FA-B, individuals HSC230 and EUFA178. HeLa cells and a lymphoblastoid cell line from a normal individual (WT) were analyzed as controls. The asterisk indicates a nonspecific band. (b) Genomic structure of FAAP95 with ten exons. The approximate locations of the translation start in exon 3 and the bipartite NLS consensus sequence are indicated. The nature and approximate locations of the mutations found in four individuals with Fanconi anemia are also indicated. Drawing is not to scale. (c) Complementation of the cellular Fanconi anemia phenotype after transfection with cDNA encoding FAAP95. Data are corrected for cellular sensitivity to MMC. The data shown are representative of several independent experiments with the same results. WT, wild-type. (d) Complementation of defective monoubiquitination of FANCD2 by transfecting cDNA encoding FAAP95 into the indicated cell lines from individuals with FA-B. a

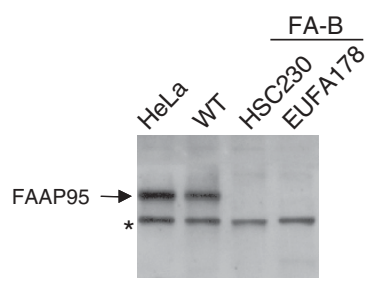

b

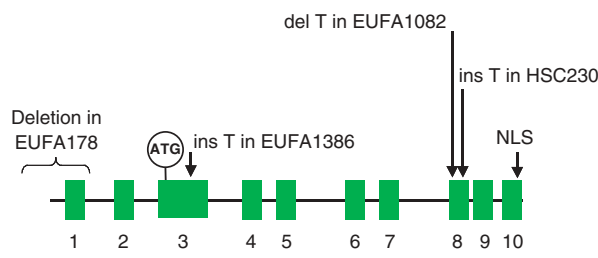

d

C

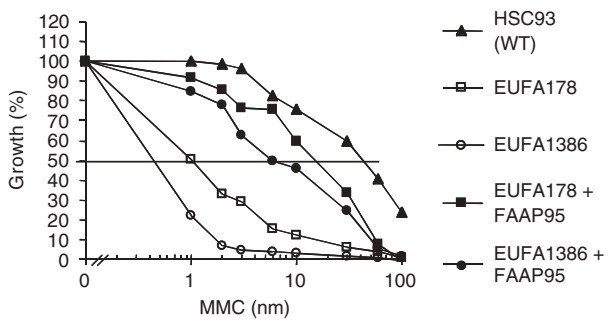

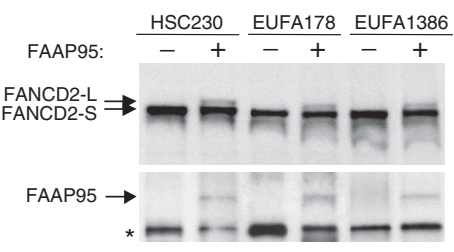

biallelically ${ }^{13,14}$. To examine whether FANCB escapes X inactivation, we assessed the methylation status of the gene, which is known to be correlated with silencing ${ }^{15}$.

We analyzed the methylation state of a CpG island in FANCB using the methylation-sensitive restriction enzyme HhaI (Fig. 6a). Without Hha I digestion, the amplified fragment showed mainly the wild-type allele (Fig. 6b). As the polymorphism is situated in a CpG island, the asymmetry of this PCR was presumably caused by a higher amplification efficiency of the unmethylated sequence as compared with the methylated sequence. After HhaI digestion, however, only the mutated allele was amplified, indicating that this allele was inactive (methylated), whereas the wild-type allele was completely active (unmethylated). In addition, direct RT-PCR amplification of mRNA from fibroblasts of individual EUFA1387 (mother of individual EUFA1386) yielded product from only the wild-type allele (Fig. 6c). These results indicate that FANCB is subject to total inactivation by methylation and that inactivation seems to be completely skewed towards the mutated allele.

To examine whether there was skewed $\mathrm{X}$ inactivation in other tissues of the mother, we analyzed the methylation status of the androgen receptor gene $(A R)$ promoter using a highly polymorphic CAG repeat. $A R$ is located on Xq11.2-12, $40 \mathrm{cM}$ from $F A N C B$, and is subject to $\mathrm{X}$ inactivation ${ }^{16}$. Results showed that $\mathrm{X}$ inactivation was skewed towards the mutant chromosome in blood and skin fibroblasts of the mother. We observed a similar situation in cells from both female carriers in the family of individual EUFA178 (Fig. 6d). The lack of a correlation with the mutant chromosome seen in individual EUFA181 was explained by a single recombination event between $F A N C B$ and $A R$ (Supplementary Fig. 4 online).

The extreme under-representation of somatic cells expressing the mutated FANCB allele in these three female carriers is not completely unexpected. Individuals with Fanconi anemia are typically growth retarded, and cultured Fanconi anemia cells are prone to apoptosis ${ }^{17}$. Thus, Fanconi anemia cells in a mosaic individual probably have a proliferative disadvantage. Because $\mathrm{X}$ inactivation takes place early in embryogenesis, selection over many subsequent cell generations may lead to almost complete overgrowth by normal cells. The female carriers studied (aged 12, 29 and 43 y) were healthy and showed no Fanconi anemia-like symptoms, and their T cells responded normally to challenge with MMC without any sign of mosaicism (data not a

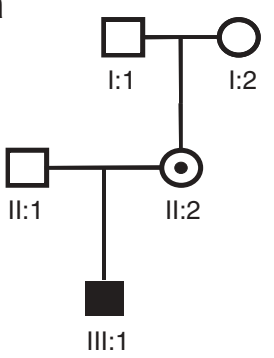

EUFA1386 b

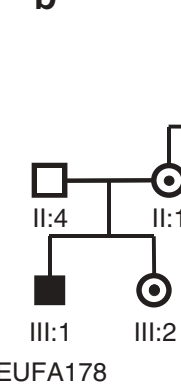

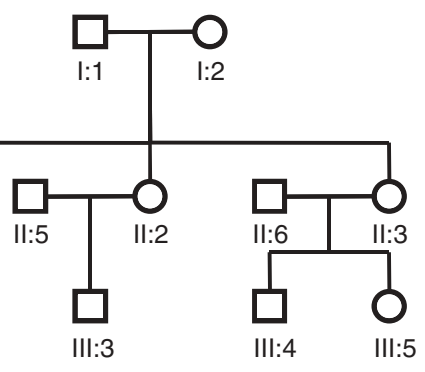

C

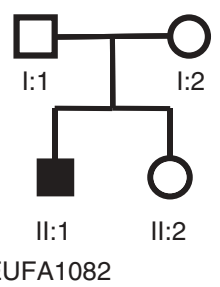

d

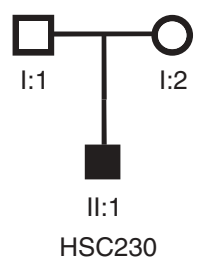

Figure 4 Pedigrees of the four families with FA-B. (a) Family EUFA1386; the proband is individual III:1, and individual II:2 (also called individual EUFA1387) is a carrier. (b) Family EUFA178; the proband is individual III:1, individuals III:2 (EUFA179) and II:1 (EUFA181) are carriers and individuals $\mathrm{II}: 3$ and III:5 are possible carriers. (c) Family EUFA1082; the proband is individual II:1. (d) Family HSC230; the proband is individual II:1. Filled symbols, individuals with FA-B; dotted symbols, carriers of a FANCB mutation. 
a

Wild-type
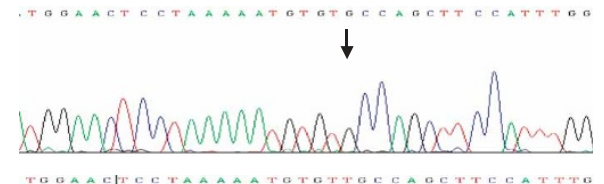

EUFA1386
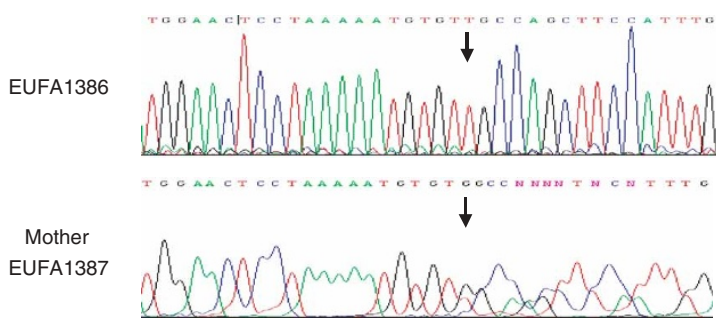

b

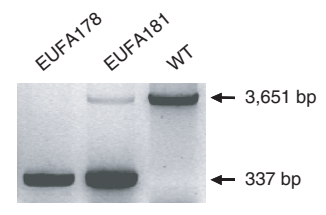

shown). We cannot, however, exclude the possibility that a few Fanconi anemia-like cells are present in these carriers. Because such 윽 cells are thought to be prone to malignant transformation (as in individuals affected with Fanconi anemia), monitoring such individuals for the occurrence of malignancies should be considered.
Figure 5 Hemizygosity and heterozygosity of mutations in FANCB. (a) Partial sequence of exon 8 of $F A N C B$ in a normal individual (wild-type), individual EUFA1386 and his mother, individual EUFA1387, showing the 811 insT mutation (arrows) in a hemizygous (EUFA1386) and heterozygous (EUFA1387) state. (b) A deletion of 3,314 bp spanning the promoter region and exon 1 is present in individual EUFA178 and his mother, individual EUFA181, generating a 337-bp product; by contrast, a control individual (WT) does not show this mutation, leading to a 3,651 bp product.

Similarly skewed $\mathrm{X}$ inactivation has been observed in some other $\mathrm{X}$-linked diseases, such as fragile $\mathrm{X}$ and Wiskott-Aldrich syndromes; however, cases have been described in which $\mathrm{X}$ inactivation is skewed towards the wild-type allele, causing symptoms of disease in female carriers $^{18,19}$. In female carriers of the FANCB mutation, there may be a strong selective force against the occurrence of such a situation. But the predictive value of our data is limited because we studied only three cases. Therefore, features of disease in carriers of the FANCB mutation cannot yet be excluded. In cases that are diagnosed in the future, assessment of skewed $\mathrm{X}$ inactivation is recommended, preferably in various tissues, as reported here.

Identification of an X-linked gene associated with Fanconi anemia has important implications for the diagnosis and genetic counseling of families in which only male children are affected with the disease. Such families should be tested to determine if they are affected with FA-B, although it is rare. In families with FA-B, mutation a

Inactive $X$ (methylated)

Active $X$

(unmethylated)

Inactive X (methylated)

Active $\mathrm{X}$

(unmethylated)

Inactive $\mathrm{X}$ (methylated)

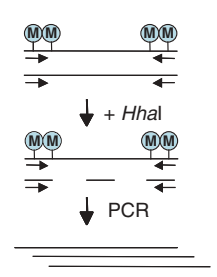

b

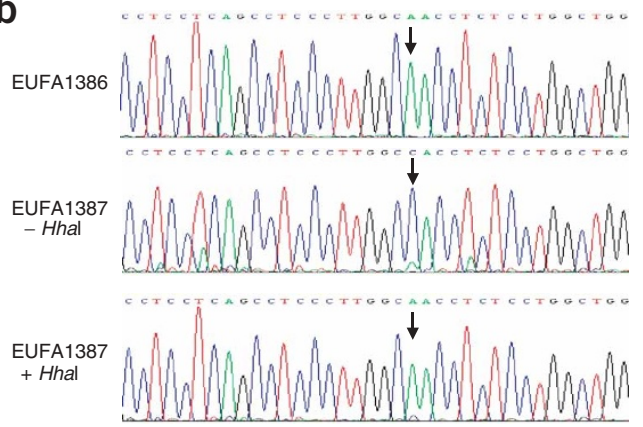

C

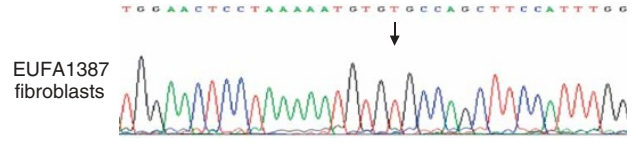

Fibroblasts
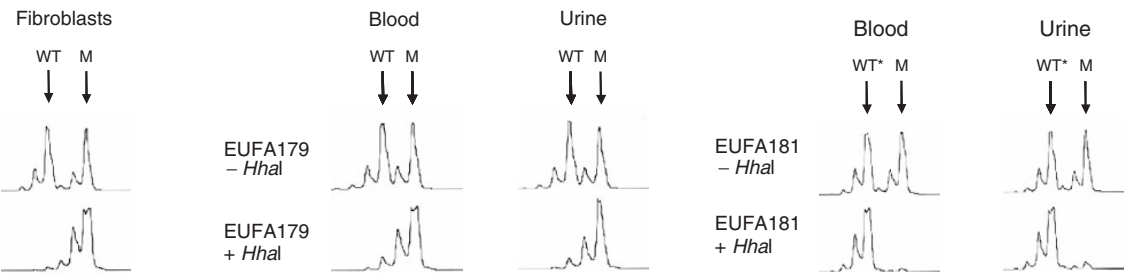

Figure 6 FANCB is subject to $\mathrm{X}$ inactivation that is skewed towards the mutated allele in heterozygous carriers of the $F A N C B$ mutation. (a) Strategy for detecting the methylated (inactive) allele of $F A N C B$ and $A R$. CpG islands are subject to methylation through the process of $\mathrm{X}$ inactivation. $A R$ has two CpG islands, whereas FANCB has only one. Digestion with the restriction enzyme Hhal, which digests only unmethylated DNA, consequently allows amplification of a fragment from a methylated (functionally inactive) allele in a PCR reaction. (b) A single-nucleotide polymorphism (A/C) present in FANCB was used to

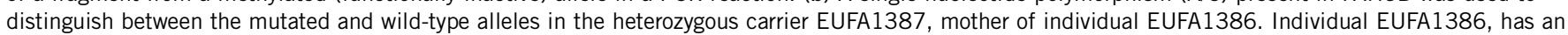
adenine at the polymorphic site, which is linked to the mutation. Individual EUFA1387 has a cytosine, with a weak adenine signal underneath. After digestion with Hhal, only the mutated FANCB allele is amplified, indicating that all wild-type FANCB allele sequences are unmethylated. (c) RT-PCR analysis of FANCB mRNA of fibroblasts from individual EUFA1387, showing expression of only the wild-type FANCB allele. Arrow indicates the position where, in the mutant sequence, a thymine is inserted (Fig. 5). (d) The polymorphic CAG repeat in the $A R$ promoter was used to examine skewed inactivation of $F A N C B$ in the indicated tissues from mutation carriers. The $A R$ alleles are linked to the wild-type (WT) allele or the mutated (M) FANCB allele as observed in affected individuals. Hhal treatment typically left the mutation-linked allele intact, indicating the methylated (inactive) status of the chromosome containing the FANCB mutation. The apparent discrepancy in individual EUFA181 (WT*) is explained by the occurrence of single recombination event between FANCB and $A R$ (Supplementary Fig. 4 online). 
screening can identify sisters and maternal aunts as carriers of the mutation who, if they test positive, have a $50 \%$ chance of conceiving an affected son or carrier daughter, irrespective of their partners.

FANCB was proposed to be BRCA2 on the basis of a hypomorphic mutation in BRCA2 detected in the HSC230 cell line ${ }^{5}$; however, no complementation of HSC230 by BRCA2 has been reported. In addition, no BRCA2 mutation has been detected in another known FA-B cell line, EUFA178 (ref. 4). Furthermore, cells with defective or depleted BRCA2 show normal monoubiquitination of FANCD2, unlike FA-B cells, which show defective FANCD2 monoubiquitination. The identification of $F A N C B$ resolves this ambiguity.

Because only a single active copy of $F A N C B$ is present in the human genome, this gene may be a potentially vulnerable component of the cellular machinery that maintains genomic integrity. Although germline mutations in $F A N C B$ are exceedingly rare, somatic FANCB mutations are likely to occur at the average spontaneous mutation rate. This may be important, because mutational inactivation of only one FANCB allele is sufficient to generate a Fanconi anemia-like cellular phenotype. The occurrence of such Fanconi anemia-like cells may contribute to oncogenesis, similar to a previously proposed mechanism $^{20,21}$ on the basis of epigenetic silencing of FANCF in a subset of sporadic malignancies. Similar to what was suggested for individuals with FANCF-silenced tumors ${ }^{20,21}$, individuals with 을 FANCB-mutated tumors might be effectively treated with crosslinking chemotherapeutic agents such as cisplatin.

\section{METHODS}

Affected individuals and cell lines. The four individuals studied were diagnosed on the basis of clinical symptoms suggestive of Fanconi anemia (Supplementary Table 1 online), coupled with a positive result from a chromosomal breakage test using MMC or diepoxybutane as a crosslinking agent. Lymphoblastoid cell line HSC230 is the first cell line representing FA-B ${ }^{22}$. The other cell lines were derived from individuals with Fanconi anemia who had been referred to the EUFAR cell repository for genetic subtyping. Individual EUFA178 was assigned to complementation group B on the basis of cell fusion studies ${ }^{4,23}$. We grew cell lines as described ${ }^{4}$. The research was (2) carried out after approval by the Institutional Review Board (VU University Medical Center, Amsterdam) and the Research Ethics Board (The Hospital for Sick Children, Toronto), and written consent was obtained from the subjects involved.

Protein purification and analysis. We immunopurified Fanconi anemia core complex from HeLa cells with an antibody to FANCA as described ${ }^{3}$. We identified FAAP95 by liquid chromatography and mass spectrometry analysis; the mass data are available on request. We generated a polyclonal rabbit antibody to FAAP95 by using a chimeric protein containing a region of FAAP95 (residues 403-485) fused to maltose-binding protein as an immunogen. This fusion protein was expressed and purified from Escherichia coli in accordance with the manufacturer's protocols (New England Biolabs) and used for both immunization and purification of the antibody. The immunoprecipitation conditions were previously described ${ }^{7}$. We prepared nuclear and cytoplasmic extracts as described ${ }^{3}$. Owing to its low abundance, FAAP95 cannot be detected by direct immunoblotting analysis of the cell extract. The main polypeptides in the nuclear extract (or total cell lysate) recognized by the FAAP95 antibody (Fig. 1b,c and Fig. 2a) are irrelevant proteins that crossreact with the antibody. FAAP95 was detected by immunoprecipitation-coupled western-blot analysis. Immunoisolation of the Fanconi anemia core complex proteins from 293 cells stably expressing Flag-tagged FANCL using the Flag antibody (Fig. 1c) has been described ${ }^{7}$. We included a mock immunoisolation using the parental 293 cell line as a control.

Cloning of FANCB and construction of vectors. We purchased an IMAGE clone (ID 6013939) encoding the hypothetical protein FLJ34064 from Invitrogen. Sequencing showed that this clone lacks $\sim 120$ nucleotides including the stop codon from the $\mathrm{C}$ terminus of the open reading frame. We amplified the missing sequence from a human cDNA library by PCR and assembled the cDNA with a full-length open reading frame by standard molecular biology techniques. The cDNA was subcloned into a mammalian expression vector, pCEP4 (Invitrogen), for complementation studies.

Transfection and complementation. We transfected HeLa cells using siRNA oligonucleotides as described ${ }^{3}$. The FAAP95 siRNA oligonucleotide comprised a 19-nucleotide target sequence (5'-UGUACUCAAGAGCCUUCAA- $\left.3^{\prime}\right)$ and was purchased from Dharmacon Research. Individual cell lines HSC230, EUFA178 and EUFA1386 were transfected with pCEP4 vector containing $F A N C B$ CDNA and selected on medium containing hygromycin at $0.1 \mathrm{mg}$ $\mathrm{ml}^{-1}$. Crosslinker sensitivity was determined in a MMC-induced growth inhibition assay, as described ${ }^{4}$. For determination of FANCD2 monoubiquitination, we treated the cells with hydroxyurea $(1 \mathrm{mM})$ for $24 \mathrm{~h}$ before collection. We confirmed that hydroxyurea induced higher levels of monoubiquitination than did MMC in the complemented cell lines.

Amplification of FANCB sequences. We isolated genomic DNA from whole blood, fibroblasts or urine samples from individuals and family members using a Qiagen Blood mini kit. For HSC230, HSC231, EUFA178 and EUFA1386, only a lymphoblastoid cell line was available. The PCR reactions for amplification of all exons of FANCB were done on 200 ng of genomic DNA using Platinum Taq polymerase (Invitrogen). To determine the deletion in EUFA178, we carried out long-range PCR on $100 \mathrm{ng}$ of genomic DNA using the Elongase system (Invitrogen).

Sequencing of FANCB. We purified PCR products by SAP/EXO treatment (Amersham), used in accordance with the manufacturer's instructions. We prepared sequencing reactions with $10 \mathrm{pmol}$ of primer and a Big Dye terminator cycle sequencing kit (Applied Biosystems) and carried them out on a GeneAmp PCR system 9700 (Applied Biosystems). Samples were analyzed on an ABI 3730 DNA Analyzer (Applied Biosystems).

FANCB inactivation. We amplified exon 1 of FANCB from Hhal-treated or untreated genomic DNA isolated from blood in a PCR reaction and sequenced it as described above. To distinguish between the two alleles, we used a polymorphism just upstream of this exon, for which individual EUFA1387 was heterozygous. RNA was isolated from fibroblasts of individual EUFA1387 with an RNeasy kit (Qiagen), and cDNA was prepared by using 10 pmol of oligo- $\mathrm{dT}_{20}$ primers and Superscript II RT polymerase (Invitrogen). The PCR reactions for amplification of FANCB were done on $60 \mathrm{ng}$ of cDNA by using Platinum Taq polymerase (Invitrogen) and sequenced as described above.

$\mathrm{X}$ inactivation. To evaluate $\mathrm{X}$ inactivation and subsequent skewing, we used the PCR protocol described for $A R^{24}$. We used 30 units of the methylation-specific enzyme Hha I to digest $1 \mu \mathrm{g}$ of genomic DNA isolated from blood, urine or fibroblasts in an overnight incubation at $37^{\circ} \mathrm{C}$. We used DNA treated the same way, but without the restriction enzyme, as a negative control. We then purified the digested and control DNAs using a PCR purification kit (Qiagen) and eluted them in $30 \mu \mathrm{l}$ of water purified by MilliQ. We then used 85-165 ng of DNA in a PCR reaction to amplify the polymorphic repeat sequence in $A R$. PCR products were diluted and mixed with Genescan-TAMRA 500 size markers (Applied Biosystems) and then analyzed on an ABI 310 DNA Analyzer (Applied Biosystems).

VNTR marker analysis. We genotyped the family of individual EUFA178 with fluorescently labeled microsatellite markers between and flanking FANCB and $A R$ and separated products by electrophoresis on an ABI 310 DNA analyzer (Applied Biosystems) $^{25}$. We used the markers DXS987, DXS9902, DXS1068, DXS1003, DXS7132, DXS6800, DXS6789 and DXS1047, which cover the region from 14.0 to $127.8 \mathrm{Mbp}$ that encompasses almost the whole X chromosome.

Note: Supplementary information is available on the Nature Genetics website.

\section{ACKNOWLEDGMENTS}

We thank the individuals and families with Fanconi anemia for contributing to this study, R. Dietrich for collecting blood and tissue samples, M. Buchwald for providing the HSC230 and HSC231 cell lines, N. Sherman for mass spectrometry analysis, J. Steltenpool and A.B. Oostra for technical assistance, R. Vervenne for suggestions, H. Willard and Q. Waisfisz for communicating unpublished results, 
D. Schlessinger for critically reading the manuscript and the National Cell Culture Center for providing cells. Financial support was from the Dutch Cancer Society, The Netherlands Organization for Health Research and Development, the FA Research Fund Inc., the Ellison medical Foundation, the US National Institutes of Health and the Deutsche FA Hilfe e.V.

\section{COMPETING INTERESTS STATEMENT}

The authors declare that they have no competing financial interests.

Received 25 June; accepted 17 September 2004

Published online at http://www.nature.com/naturegenetics/

1. Joenje, H. \& Patel, K.J. The emerging genetic and molecular basis of Fanconi anemia. Nat. Genet. 2, 446-457 (2001).

2. D'Andrea, A.D. \& Grompe, M. The Fanconi anaemia/BRCA pathway. Nat. Rev. Cancer 3, 23-34 (2003).

3. Meetei, A.R. et al. A multiprotein nuclear complex connects Fanconi anemia and Bloom syndrome. Mol. Cell. Biol. 23, 3417-3426 (2003).

4. Levitus, M. et al. Heterogeneity in Fanconi anemia: evidence for two new genetic subtypes. Blood 103, 2498-2503 (2004).

5. Howlett, N.G. et al. Biallelic inactivation of BRCA2 in Fanconi anemia. Science 297, 606-609 (2002).

6. Garcia-Higuera, I. et al. Interaction of the Fanconi anemia protein and BRCA1 in a common pathway. Mol. Cell 7, 249-262 (2001).

7. Meetei, A.R. et al. A novel ubiquitin ligase is deficient in Fanconi anemia. Nat. Genet. 35, 165-170 (2003).

8. Bruun, D. et al. siRNA depletion of BRCA1, but not BRCA2, causes increased genome instability in Fanconi anemia cells. DNA Repair 2, 1007-1013 (2003).

9. Jegalian, K. \& Page, D.C. A proposed path by which genes common to mammalian $X$ and $Y$ chromosomes evolve to become X inactivated. Nature 394, 776-780 (1998).

10. Monk, M. \& Harper, M.I. Sequential X chromosome inactivation coupled with cellular differentiation in early mouse embryos. Nature 281, 311-313 (1979).

11. Lyon, M.F. Gene action in the X-chromosome of the mouse (Mus musculus L). Nature 190, 372-373 (1961).
12. Russell, L.B. Genetics of mammalian sex chromosomes. Science 133, 1795-1803 (1961).

13. Brown, C.J. \& Greally, J.M. A stain upon the silence: genes escaping $X$ inactivation. Trends Genet. 19, 432-438 (2003).

14. Carrel, L., Cottle, A.A., Goglin, K.C. \& Willard, H.F. A first-generation X-inactivation profile of the human X chromosome. Proc. Natl. Acad. Sci. USA 96, 14440-14444 (1999).

15. Lee, J.T. Molecular links between X-inactivation and autosomal imprinting: X-inactivation as a driving force for the evolution of imprinting? Curr. Biol. 13, 242-254 (2003).

16. Allen, R.C., Zoghbi, H.Y., Moseley, A.B., Rosenblatt, H.M. \& Belmont, J.W. Methylation of Hpall and Hhal sites near the polymorphic CAG repeat in the human androgenreceptor gene correlates with X chromosome inactivation. Am. J. Hum. Genet. 51, 1229-1239 (1992).

17. Cumming, R.C. et al. Fanconi anemia group C protein prevents apoptosis in hematopoietic cells through redox regulation of GSTP1. Nat. Med. 7, 814-820 (2001).

18. Heine-Suner, D. et al. Fragile-X syndrome and skewed X-chromosome inactivation within a family: a female member with complete inactivation of the functional $X$ chromosome. Am. J. Med. Genet. 122A, 108-114 (2003).

19. Andreu, N. et al. Wiskott-Aldrich syndrome in a female with skewed X-chromosome inactivation. Blood Cells Mol. Dis. 31, 332-337 (2003).

20. Taniguchi, T. et al. Disruption of the Fanconi anemia-BRCA pathway in cisplatinsensitive ovarian tumors. Nat. Med. 9, 568-574 (2003).

21. Tischkowitz, M. et al. Bi-allelic silencing of the Fanconi anaemia gene FANCF in acute myeloid leukaemia. Br. J. Haematol. 123, 469-471 (2003).

22. Strathdee, C.A., Duncan, A.M. \& Buchwald, M. Evidence for at least four Fanconi anaemia genes including FACC on chromosome 9. Nat. Genet. 1, 196-198 (1992).

23. de Winter, J.P. et al. The Fanconi anemia protein FANCF forms a nuclear complex with FANCA, FANCC and FANCG. Hum. Mol. Genet. 9, 2665-2674 (2000).

24. Tanaka, M. et al. Evidence of the monoclonal composition of human endometrial epithelial glands and mosaic pattern of clonal distribution in luminal epithelium. Am. J. Pathol. 163, 295-301 (2003).

25. Fanconi Anaemia/Breast Cancer Consortium. Positional cloning of the Fanconi anemia group A gene. Nat. Genet. 14, 324-328 (1996). 